\title{
Pneumonia: 3 Days of Antibiotics for Uncomplicated Course
}

- CLINICAL QUESTION: In patients hospitalized for treatment of community-acquired pneumonia, can treatment be stopped after 3 days if the patient has substantially improved?

- BOTTOM LINE: Dogma successfully challenged: In patients who respond well to initial treatment, stopping antibiotic therapy after 3 days is just as effective as continuing treatment for the standard 8 days. (LOE $=1 b)$

REFERENCE: el Moussaoui R, de Borgie CA, van den Broek $\mathrm{P}$, et al. Effectiveness of discontinuing antibiotic treatment after three days versus eight days in mild to moderate-severe community acquired pneumonia: randomised, double blind study. BMJ 2006;332:13551358.

- STUDY DESIGN: Randomized controlled trial (double-blinded)

FUNDING: Industry

ALLOCATION: Uncertain

SETTING: Inpatient (any location)

SYNOPSIS: The treatment of pneumonia for 7 days to 10 days is based on tradition, not scientific evidence. The researchers conducting this study challenged the status quo by enrolling 119 adults with mild to moderate-severe community-acquired pneumonia with a severity index score of 110 or less. On admission, all patients were started on intravenous amoxicillin, the preferred empirical treatment in the Netherlands. After 72 hours of treatment, patients who showed improvement in symptoms, had a temperature of less than $38 \mathrm{C}$, and could take oral drugs were randomized to treatment with placebo or amoxicillin $750 \mathrm{mg} 3$ times daily for 5 days. Using modified intention-to-treat analysis, after 10 days $89 \%$ of patients in both groups were clinically cured. In follow-up at 28 days, clinical cure rates were also similar between the 2 approaches, as was bacteriologic success and radiologic success. This study was designed to find a difference in success rates of at least $10 \%$. There are a couple of notable limitations to this study. First, the patients in the short-treatment group had a median age of 54 years compared with 60 years in the 8 -day group, and these younger patients may be more likely to respond to the short course, thus skewing the results. Second, the study was conducted in the Netherlands, where resistance patterns may be different than in other countries. Finally, the study was conducted in 9 hospitals over 3 years, which works out to less than 5 patients per hospital per year recruited into the study. Given the imbalance in age and this sparse representation, these patients could be highly selected and not representative of the typical patient admitted to a community hospital. 